Document downloaded from:

http://hdl.handle.net/10251/140191

This paper must be cited as:

Ferreyra, DE.; Orquera, V.; Thome, N. (10-2). A weak group inverse for rectangular matrices. Revista de la Real Academia de Ciencias Exactas Físicas y Naturales Serie A Matemáticas. 113(4):3727-3740. https://doi.org/10.1007/s13398-019-00674-9

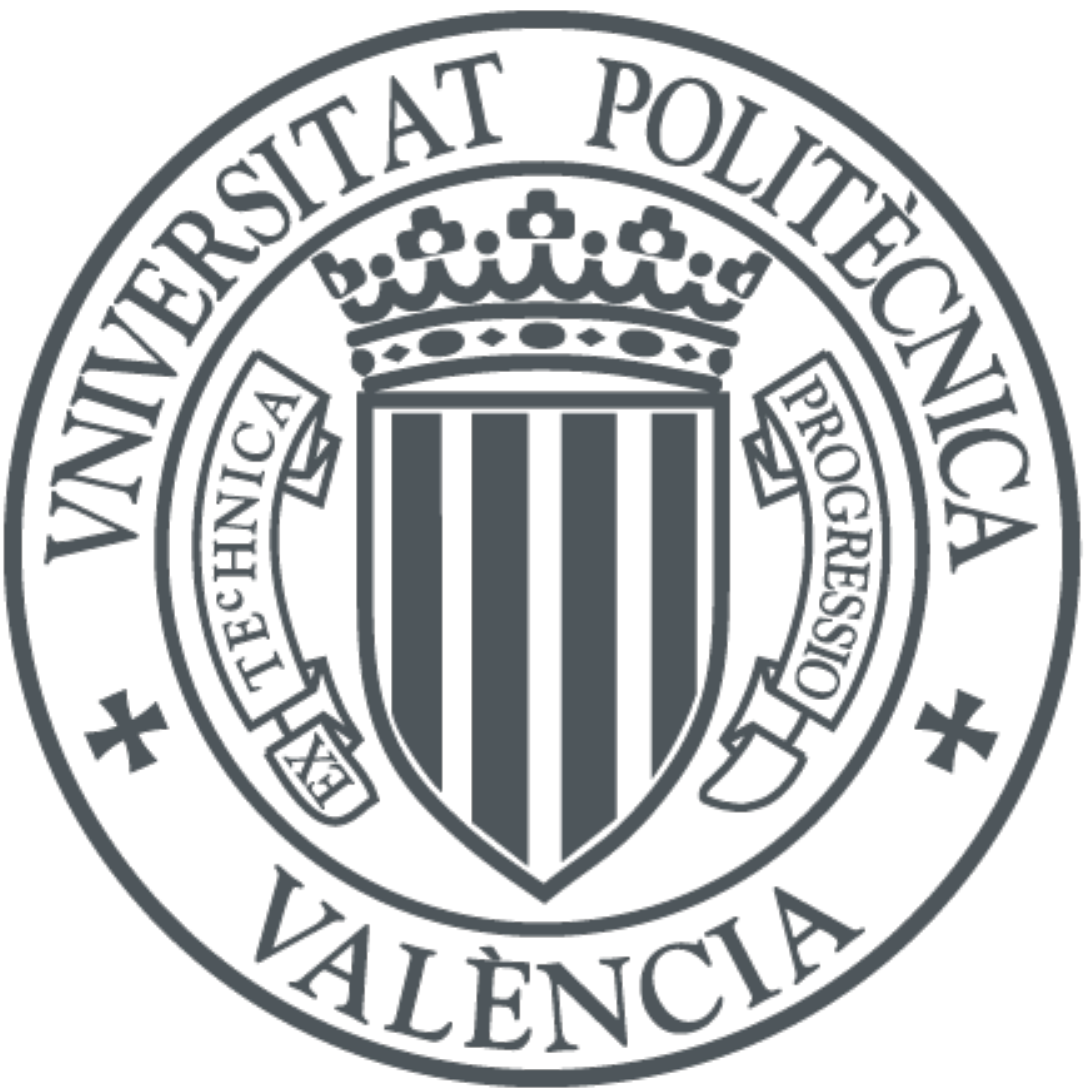

The final publication is available at

https://doi.org/10.1007/s13398-019-00674-9

Copyright Springer-Verlag

Additional Information 


\title{
A weak group inverse for rectangular matrices
}

\author{
D.E. Ferreyra · V. Orquera · N. Thome
}

Received: date / Accepted: date

\begin{abstract}
In this paper, we extend the notion of weak group inverse to rectangular matrices (called $W$-weighted WG inverse) by using the weighted core EP inverse recently investigated. This new generalized inverse also generalizes the well-known weighted group inverse given by Cline and Greville. In addition, we give several representations of the $W$-weighted WG inverse, and derive some characterizations and properties.
\end{abstract}

Keywords Generalized inverses · weighted weak group inverse · weighted core EP inverse · weighted Drazin inverse

Mathematics Subject Classification (2010) 15A09

\section{Introduction}

In 1920, E.H. Moore introduced the general reciprocal trying to find a matrix that plays the role of the inverse of a singular or a nonsquare matrix. For a given

An earlier version of this paper was presented at the Conference "Linear Algebra, Matrix Analysis and Applications. ALAMA2018", held in Sant Joan d'Alacant on May/June 2018.

First author was partially supported by UNRC (grant PPI 18/C472) and CONICET (grant PIP 112-201501-00433CO). Third author was partially supported by Ministerio de Economía, Industria y Competitividad of Spain (Grants DGI MTM2013-43678-P and Red de Excelencia MTM2017-90682-REDT).

D.E. Ferreyra

Universidad Nacional de Río Cuarto, FCEFQyN, RN 36 Km 601, Río Cuarto, 5800, Córdoba, Argentina.

E-mail: deferreyra@exa.unrc.edu.ar

V. Orquera

Universitat Politécnica de Valéncia, Valencia, 46022, Spain.

E-mail: vaor2@posgrado.upv.es

N. Thome

Instituto Universitario de Matemática Multidisciplinar, Universitat Politècnica de València, 46022, Valencia, Spain.

E-mail: njthome@mat.upv.es 
complex rectangular matrix $A$, this (geometric) definition employs the orthogonal projector onto the range of $A$ and that onto the range of the conjugate transpose of $A$. It had not success (mainly, due to its complicated notation) until R. Penrose considered in 1955 four (algebraic) matrix equations that characterize the same inverse, nowadays known as the Moore-Penrose inverse [21]. On the other hand, three years later, a new generalized inverse was introduced by M.P. Drazin in [9], having into account spectral aspects in the setting of complex matrices. Many properties and applications were developed from that moment until today. For instance, generalized inverses are used to solve linear systems (specially, to solve least squares problems), matrix equations, singular difference and differential equations, singular control linear systems, to study public key crypto system design $[1,5,13,14]$, etc. In particular, generalized inverses appear as a useful tool in areas such as Markov chains [5,14], Cryptography [13], Chemical equations [22], Robotics [4,8], Coding theory [23], Optimization theory [12], Computer graphic [16], etc.

The set of all $m \times n$ complex matrices will be denoted by $\mathbb{C}^{m \times n}$. For $A \in \mathbb{C}^{m \times n}$, the symbols $A^{*}, A^{-1}, \operatorname{rk}(A), \mathcal{N}(A)$, and $\mathcal{R}(A)$ will denote the conjugate transpose, the inverse (whenever it exists), the rank, the kernel, and the range space of $A$, respectively. Moreover, $I_{n}$ will refer to the $n \times n$ identity matrix.

Let $A \in \mathbb{C}^{m \times n}$. The Moore-Penrose inverse of $A$, denoted by $A^{\dagger}$, is defined as the unique matrix $X \in \mathbb{C}^{n \times m}$ satisfying the following four equations:

$$
\text { (1) } A X A=A, \quad(2) X A X=X, \quad(3)(A X)^{*}=A X, \quad(4)(X A)^{*}=X A \text {. }
$$

A matrix $X \in \mathbb{C}^{n \times m}$ that satisfies the unique equality $A X A=A$ is called an inner inverse (or $\{1\}$-inverse) of $A$ (these inverses can be used as a system solver), and a matrix $X \in \mathbb{C}^{n \times m}$ that satisfies the unique equality $X A X=X$ is called an outer inverse (or $\{2\}$-inverse) of $A$. The Moore-Penrose inverse is used to represent the orthogonal projectors $P_{A}:=A A^{\dagger}$ and $Q_{A}:=A^{\dagger} A$ onto $\mathcal{R}(A)$ and onto $\mathcal{R}\left(A^{*}\right)$, respectively.

For a given complex square matrix $A$, the index of $A$, denoted by $\operatorname{Ind}(A)$, is the smallest nonnegative integer $k$ such that $\mathcal{R}\left(A^{k}\right)=\mathcal{R}\left(A^{k+1}\right)$. We observe that the index of a nonsingular matrix $A$ is 0 , and by convention, the index of the null matrix is 1 .

We recall that the Drazin inverse of $A \in \mathbb{C}^{n \times n}$ is the unique matrix $X \in \mathbb{C}^{n \times n}$ such that

$$
\left(1^{k}\right) X A^{k+1}=A^{k}, \quad \text { (2) } X A X=X, \quad \text { (5) } A X=X A,
$$

where $k=\operatorname{Ind}(A)$. It always exists, it is unique, and will be denoted by $A^{d}$. If $A \in \mathbb{C}^{n \times n}$ satisfies $\operatorname{Ind}(A) \leq 1$, then the Drazin inverse of $A$ is called the group inverse of $A$ and is denoted by $A^{\#}$.

The core inverse was introduced by O. Baksalary and G. Trenkler in [2]. For a given matrix $A \in \mathbb{C}^{n \times n}$, the core inverse of $A$ is the unique matrix $X \in \mathbb{C}^{n \times n}$ defined by the conditions $A X=P_{A}$ and $\mathcal{R}(X) \subseteq \mathcal{R}(A)$. In case that such a matrix $X$ exists, it is denoted by $A^{\boxplus}$. Moreover, it was proved that $A$ is core invertible if and only if $\operatorname{Ind}(A) \leq 1$.

Several generalizations of the core inverse were recently introduced for $n \times n$ complex matrices, namely B-T inverses (O. Baksalary and G. Trenkler), DMP inverses (S. Malik and N. Thome), and core EP inverses (K. Manjunatha Prasad and K.S. Mohana). Let $A \in \mathbb{C}^{n \times n}$ with $\operatorname{Ind}(A)=k$. The DMP inverse of $A$ is 
given by $A^{d, \dagger}=A^{d} A A^{\dagger}[15]$. The B-T inverse of $A$ is $A^{\diamond}=\left(A P_{A}\right)^{\dagger}$ [3]. The core EP inverse of $A$ is $A^{\oplus}=A^{k}\left(\left(A^{*}\right)^{k} A^{k+1}\right)^{\dagger}\left(A^{*}\right)^{k}$ [17]. The uniqueness of all of them is clear as well as their existence for arbitrary index.

Let $A \in \mathbb{C}^{m \times n}$ and $0 \neq W \in \mathbb{C}^{n \times m}$. R.E. Cline and T.N. Greville [6] extended the Drazin inverse from square matrices to rectangular ones and was called the weighted Drazin inverse; it is given by $A^{d, W}=\left[(A W)^{d}\right]^{2} A=A\left[(W A)^{d}\right]^{2}$. In [10], the authors defined the weighted core EP inverse which will be crucial in this paper. It is a kind of generalized inverse defined for rectangular matrices by extending the concept of core EP inverse known for square matrices. Recently, the DMP inverse was extended from square matrices to rectangular matrices by L.S. Meng in [19] and was called the $W$-weighted DMP inverse; it is given by $A^{d, \dagger, W}=$ $W A^{d, W} W P_{A}$. In addition, a new generalized inverse was investigated in [18] by M. Mehdipour and A. Salemi. In this case, the matrix $A^{c, \dagger}=Q_{A} A^{d} P_{A}$, is called the CMP inverse of $A$. In order to extend the CMP inverse from the square to the rectangular case, the weighted CMP inverse was defined by D. Mosić in [20] as $A^{c, \dagger, W}=Q_{A} W A^{d, W} W P_{A}$.

Combining the two properties $A X A=A$ and $A X=X A$ satisfied by the group inverse, recently, H. Wang and J. Chen defined other generalized inverse for square matrices in [25] by using the core EP inverse. In this case, the unique matrix $X \in \mathbb{C}^{n \times n}$ satisfying

$$
A X^{2}=X \quad \text { and } \quad A X=A^{\oplus} A,
$$

is called the weak group inverse of $A$ and is denoted by $A^{\circledR}$.

The main aim of this paper is to consider a weight $W$ conformable to a given rectangular matrix $A$ such that $A W$ (or $W A$ ) has arbitrary index, $A W$ and $W A$ are well defined and then investigate the weighted weak group inverse. While the formulas that generalize the weak group inverse to the weighted weak group inverse seem to be a natural extension (see Definition 3), we would like to highlight that the methods used in our proofs require a much more deep understanding of this issue because Theorem 5, Theorem 7, Theorem 8, and so on, could not be obtained unless we take advantage of definition and properties of the weighted core EP inverse recently introduced (see [10]).

Below, we add a glossary with the main definitions and notations related to the different generalized inverses used throughout the paper:
1.- $A^{\dagger} \quad$ Moore-Penrose inverse
2.- $A^{d} \quad$ Drazin inverse
3.- $A^{\#} \quad$ Group inverse
4.- $A^{\oplus} \quad$ Core inverse
5.- $A^{\diamond} \quad$ B-T inverse
6.- $A^{\oplus} \quad$ Core EP inverse
7.- $A^{d, \dagger} \quad$ DMP inverse
8.- $A^{c, \dagger} \quad$ CMP inverse
9.- $A^{\circledR} \quad$ Weak group inverse
10.- $A^{W, d}$ Weighted Drazin inverse
11.- $A^{\oplus, W}$ Weighted core EP inverse
12.- $A^{d, \dagger, W} \mathrm{~W}$-weighted DMP inverse
13.- $A^{c, \dagger, W}$ Weighted CMP inverse

This paper is organized as follows. Section 2 recalls the weak group inverse and the weighted core EP inverse of a matrix which are computed by means of the core 
EP decomposition and the weighted core EP decomposition, respectively. We also obtain a new way to compute the weighted Drazin inverse and, particularly, for the computation of the weighted group inverse. Section 3 introduces and investigates the weighted weak group inverse. This new generalized inverse is presented as the unique solution of a system of matrix equations. We exhibit some examples to show that this new inverse differs from others well-known inverses. Also, an explicit formula for its computation is provided. Section 4 analyses some properties, representations, and characterizations for weighted weak group inverses. In addition, some relationships to the well-known inverses are given.

\section{Preliminary results}

In this section, we present some background and then derive some new results for the weighted Drazin (group) inverse. In [24], H. Wang introduced the core EP decomposition. It was proved that for every nonzero matrix $A \in \mathbb{C}^{n \times n}$ with $\operatorname{Ind}(A)=k \geq 1$, there exist unique matrices $A_{1}, A_{2} \in \mathbb{C}^{n \times n}$ such that $A=A_{1}+A_{2}$ satifying $\operatorname{Ind}\left(A_{1}\right) \leq 1, A_{2}^{k}=0$, and $A_{1}^{*} A_{2}=A_{2} A_{1}=0$ ([24, Theorem 2.1 , Theorem 2.4]). Moreover, there exists a unitary matrix $U \in \mathbb{C}^{n \times n}$ such that $A$ can be represented as the sum of

$$
A_{1}=U\left[\begin{array}{ll}
T & S \\
0 & 0
\end{array}\right] U^{*} \quad \text { and } \quad A_{2}=U\left[\begin{array}{ll}
0 & 0 \\
0 & N
\end{array}\right] U^{*}
$$

where $T$ is nonsingular, $\operatorname{rk}(T)=\operatorname{rk}\left(A^{k}\right)$, and $N$ is nilpotent of index $k$. This representation of $A$ is called the core EP decomposition of $A$.

Based on decomposition (2) for $A, \mathrm{H}$. Wang proved that the core EP inverse of $A$ has the form

$$
A^{\oplus}=U\left[\begin{array}{cc}
T^{-1} & 0 \\
0 & 0
\end{array}\right] U^{*} .
$$

Similarly, in [11, Theorem 3.8], a new representation for Drazin matrices by using the core EP decomposition was obtained.

Theorem 1 Let $A \in \mathbb{C}^{n \times n}$ be a matrix of index $k \geq 1$ written as in (2). Then

$$
A^{d}=U\left[\begin{array}{cc}
T^{-1} & \widetilde{T} \\
0 & 0
\end{array}\right] U^{*}, \quad \text { where } \widetilde{T}=\sum_{j=0}^{k-1} T^{j-k-1} S N^{k-1-j} .
$$

In [25], after defining the weak group inverse $A^{\circledR}$ of $A$ as such matrix $X \in \mathbb{C}^{n \times n}$ that satisfies $A X^{2}=X$ and $A X=A^{\oplus} A$, it was also proved that this generalized inverse can be computed as

$$
A^{\circledR}=U\left[\begin{array}{cc}
T^{-1} & T^{-2} S \\
0 & 0
\end{array}\right] U^{*}
$$

provided that $A=A_{1}+A_{2}$ is written as in (2).

Throughout this paper, a nonzero matrix $W \in \mathbb{C}^{n \times m}$ will be fixed and used as a weight. In what follows, this weight matrix $W$ will be not explicitly mentioned. For $A \in \mathbb{C}^{n \times m}$, we notice that $A W \in \mathbb{C}^{m \times m}$ and $W A \in \mathbb{C}^{n \times n}$.

We recall the definition of the weighted Drazin inverse for rectangular matrices introduced by R.E. Cline and T.N. Greville in [6]. 
Definition 1 Let $A \in \mathbb{C}^{m \times n}$. A matrix $X \in \mathbb{C}^{m \times n}$ is a weighted Drazin inverse of $A$ if $A W X=X W A, X W A W X=X$, and $X W(A W)^{k+1}=(A W)^{k}$ with $k=$ $\max \{\operatorname{Ind}(A W), \operatorname{Ind}(W A)\}$.

This matrix $X$ always exists, is unique, and will be denoted by $X=A^{d, W}$. Moreover, the equalities

$$
A^{d, W}=A\left[(W A)^{d}\right]^{2}=\left[(A W)^{d}\right]^{2} A, \quad A^{d, W} W=(A W)^{d}, \quad W A^{d, W}=(W A)^{d},
$$

hold [6]. When $m=n$ and $W=I_{n}$ the Drazin inverse is recovered, that is, $A^{d, W}=A^{d}$. For the particular case of being $k=1$, the weighted Drazin inverse of $A$ is called the weighted group inverse of $A$ and is denoted by $A^{\#, W}$.

The following definition was introduced by D. Ferreyra, F. Levis, and N. Thome in $[10]$.

Definition 2 Let $A \in \mathbb{C}^{m \times n}$ and $k=\max \{\operatorname{Ind}(A W)$, $\operatorname{Ind}(W A)\}$. The unique matrix $X \in \mathbb{C}^{m \times n}$ such that $W A W X=P_{(W A)^{k}}$ and $\mathcal{R}(X) \subseteq \mathcal{R}\left((A W)^{k}\right)$ is called the weighted core EP inverse of $A$, and is denoted by $A^{\oplus, W}$.

When $m=n$ and $W=I_{n}$ we recover the core EP inverse, that is, $A^{\oplus, W}=A^{\oplus}$. When $k=1$ the weighted core EP inverse provides an interesting particular case that shall be called the weighted core inverse and denoted by $A^{\boxplus, W}$, that is, $A^{\oplus, W}=A^{\boxplus, W}$ for $k=1$. As far as we know, this case was not previously studied in the literature and it provides a new kind of generalized inverse defined on the class of at most 1 index matrices.

Also, in [10] the authors introduced a new decomposition, called weighted core EP decomposition, extending the core EP decomposition from square to rectangular matrices. This result establishes a simultaneous unitary block triangularization of a pair of rectangular matrices.

Theorem 2 Let $A \in \mathbb{C}^{m \times n}$ and $k=\max \{\operatorname{Ind}(A W), \operatorname{Ind}(W A)\}$. Then there exist two unitary matrices $U \in \mathbb{C}^{m \times m}, V \in \mathbb{C}^{n \times n}$, two nonsingular matrices $A_{1}, W_{1} \in \mathbb{C}^{t \times t}$, and two matrices $A_{2} \in \mathbb{C}^{(m-t) \times(n-t)}$ and $W_{2} \in \mathbb{C}^{(n-t) \times(m-t)}$ such that $A_{2} W_{2}$ and $W_{2} A_{2}$ are nilpotent of indices $\operatorname{Ind}(A W)$ and $\operatorname{Ind}(W A)$, respectively, with

$$
A=U\left[\begin{array}{cc}
A_{1} & A_{12} \\
0 & A_{2}
\end{array}\right] V^{*} \quad \text { and } \quad W=V\left[\begin{array}{cc}
W_{1} & W_{12} \\
0 & W_{2}
\end{array}\right] U^{*} .
$$

The expressions for $A$ and $W$ provided in Theorem 2 give the so called weighted core EP decomposition of the pair $\{A, W\}$.

The weighted core EP inverse of a rectangular matrix was represented by using the weighted core EP decomposition [10, Theorem 5.2]. More precisely, the weighted core EP inverse of $A \in \mathbb{C}^{m \times n}$ has the form

$$
A^{\oplus, W}=U\left[\begin{array}{cc}
\left(W_{1} A_{1} W_{1}\right)^{-1} & 0 \\
0 & 0
\end{array}\right] V^{*} .
$$

Remark 1 When $m=n$ and $W=I_{n}$, from the representations given in (3) and (8), it is easy to verify that the weighted core EP inverse and the core EP inverse are coincide. 
Now, we derive a new representation for weighted Drazin inverses by using the weighted core EP decomposition of the pair $\{A, W\}$.

Theorem 3 Let $A \in \mathbb{C}^{m \times n}$ written as in (7) with $k=\max \{\operatorname{Ind}(A W), \operatorname{Ind}(W A)\} \geq$ 1. Then

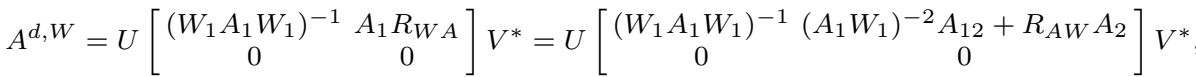

where

$$
R_{A W}=\sum_{j=0}^{k-1}\left(A_{1} W_{1}\right)^{j-k-2}\left(A_{1} W_{12}+A_{12} W_{2}\right)\left(A_{2} W_{2}\right)^{k-1-j}
$$

and

$$
R_{W A}=\sum_{j=0}^{k-1}\left(W_{1} A_{1}\right)^{j-k-2}\left(W_{1} A_{12}+W_{12} A_{2}\right)\left(W_{2} A_{2}\right)^{k-1-j}
$$

Proof We assume that the pair $\{A, W\}$ is written as in (7) in the weighted core EP decomposition. Now, we notice that the expressions

$$
A W=U\left[\begin{array}{cc}
A_{1} W_{1} & A_{1} W_{12}+A_{12} W_{2} \\
0 & A_{2} W_{2}
\end{array}\right] U^{*} \text { and } W A=V\left[\begin{array}{cc}
W_{1} A_{1} & W_{1} A_{12}+W_{12} A_{2} \\
0 & W_{2} A_{2}
\end{array}\right] V^{*},
$$

allow us to write them in the core EP decomposition as in (2). Applying Theorem 1 to both matrices $A W$ and $W A$ we obtain

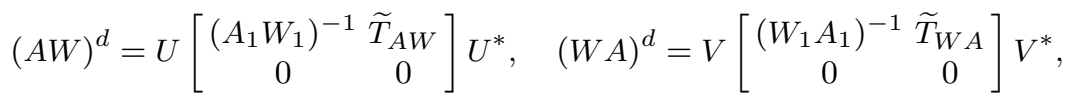

where

$$
\widetilde{T}_{A W}=\sum_{j=0}^{k-1}\left(A_{1} W_{1}\right)^{j-k-1}\left(A_{1} W_{12}+A_{12} W_{2}\right)\left(A_{2} W_{2}\right)^{k-1-j}
$$

and

$$
\widetilde{T}_{W A}=\sum_{j=0}^{k-1}\left(W_{1} A_{1}\right)^{j-k-1}\left(W_{1} A_{12}+W_{12} A_{2}\right)\left(W_{2} A_{2}\right)^{k-1-j}
$$

Now, by setting $R_{A W}:=\left(A_{1} W_{1}\right)^{-1} \widetilde{T}_{A W}$ and $R_{W A}:=\left(W_{1} A_{1}\right)^{-1} \widetilde{T}_{W A}$, the expressions for (9) follow by a simple computation by using (6).

Corollary 1 Let $A \in \mathbb{C}^{m \times n}$ written as in (7) where $\max \{\operatorname{Ind}(A W), \operatorname{Ind}(W A)\}=1$. Then

$$
\begin{aligned}
A^{\#, W} & =U\left[\begin{array}{cc}
\left(W_{1} A_{1} W_{1}\right)^{-1} & \left(A_{1} W_{1}\right)^{-2}\left(A_{12}+W_{1}^{-1} W_{12} A_{2}\right) \\
0 & 0
\end{array}\right] V^{*} \\
& =U\left[\begin{array}{cc}
\left(W_{1} A_{1} W_{1}\right)^{-1} & \left(A_{1} W_{1}\right)^{-2}\left[A_{12}+\left(A_{1} W_{1}\right)^{-1}\left(A_{1} W_{12}+A_{12} W_{2}\right) A_{2}\right] \\
0 & 0
\end{array}\right] V^{*} .
\end{aligned}
$$


3 Weighted weak group inverse: existence and uniqueness

The formula of the weak group inverse given by H. Wang and J. Chen in [25] serves for computing that inverse only for square matrices. In this section, we introduce and investigate the weighted weak group inverse for rectangular matrices $A \in \mathbb{C}^{m \times n}$ by considering a conformable weight $W \in \mathbb{C}^{n \times m}$ such that $A W$ and $W A$ are well-defined as square matrices and $A W$ having arbitrary index. Notice that the indices of $A W$ and $W A$ may differ at most in 1.

Let $W \in \mathbb{C}^{n \times m}$ be a fixed nonzero matrix and $A, B \in \mathbb{C}^{m \times n}$. We define the $W$ product of $A$ and $B$ by $A \star B=A W B$, and we denote the $W$-product of $A$ with itself $\ell$ times by $A^{\star \ell}$. It is well known that if $\|A\|_{W}=\|A\|\|W\|$ then $\left(\mathbb{C}^{m \times n}, \star,\|\cdot\|_{W}\right)$ is a Banach algebra and

$$
A^{\star \ell}=(A W)^{\ell-1} A=A(W A)^{\ell-1}, \quad \ell \in \mathbb{N},
$$

where $\|\cdot\|$ denotes any (fixed but arbitrary) matrix norm on $\mathbb{C}^{m \times n}$.

Let $0 \neq W \in \mathbb{C}^{n \times m}, A \in \mathbb{C}^{m \times n}$, and $k=\max \{\operatorname{Ind}(A W), \operatorname{Ind}(W A)\}$. We consider the matrix system given by

$$
A \star X^{\star 2}=X, \quad A \star X=A^{\oplus, W} \star A .
$$

Theorem 4 If the system (12) has a solution then it is unique.

Proof Assume that both $X_{1}$ and $X_{2}$ satisfy (12). In particular, $A \star X_{1}=A^{\oplus, W} \star A=$ $A \star X_{2}$. By using the associativity of the $W$-product we have

$X_{1}=A \star X_{1}^{\star 2}=\left(A \star X_{1}\right) \star X_{1}=A^{\oplus, W} \star A \star X_{1}=A^{\oplus, W} \star A \star X_{2}=A \star X_{2}^{\star 2}=X_{2}$.

When the unique matrix of Theorem 4 exists, it is denoted by $A^{\circledR, W}$.

Now, we establish the existence and representation of the unique solution of the system (12) by using the weighted core EP decomposition.

Theorem 5 The system (12) is always consistent and its unique solution is given by

$$
A^{\circledR, W}=U\left[\begin{array}{cc}
\left(W_{1} A_{1} W_{1}\right)^{-1} & \left(A_{1} W_{1}\right)^{-2}\left(A_{12}+W_{1}^{-1} W_{12} A_{2}\right) \\
0 & 0
\end{array}\right] V^{*},
$$

where the given matrix $A \in \mathbb{C}^{m \times n}$ is expressed as in (7).

Proof From decomposition (7) given in Theorem 2 for the pair $\{A, W\}$ and by (8) we have

$$
\begin{aligned}
& X:=\left(A^{\oplus, W}\right)^{\star 2} \star A=A^{\oplus, W} \star A^{\oplus, W} \star A=A^{\oplus, W} W A^{\oplus, W} W A \\
& =U\left[\begin{array}{cc}
\left(W_{1} A_{1} W_{1}\right)^{-1} & 0 \\
0 & 0
\end{array}\right]\left[\begin{array}{cc}
W_{1} & W_{12} \\
0 & W_{2}
\end{array}\right]\left[\begin{array}{cc}
\left(W_{1} A_{1} W_{1}\right)^{-1} & 0 \\
0 & 0
\end{array}\right]\left[\begin{array}{cc}
W_{1} A_{1} & W_{1} A_{12}+W_{12} A_{2} \\
0 & W_{2} A_{2}
\end{array}\right] V^{*} \\
& =U\left[\begin{array}{cc}
\left(W_{1} A_{1} W_{1}\right)^{-1}\left(W_{1} A_{1}\right)^{-1} & 0 \\
0 & 0
\end{array}\right]\left[\begin{array}{cc}
W_{1} A_{1} & W_{1} A_{12}+W_{12} A_{2} \\
0 & W_{2} A_{2}
\end{array}\right] V^{*} \\
& =U\left[\begin{array}{cc}
\left(W_{1} A_{1} W_{1}\right)^{-1} & \left(W_{1} A_{1} W_{1}\right)^{-1}\left(W_{1} A_{1}\right)^{-1}\left(W_{1} A_{12}+W_{12} A_{2}\right) \\
0 & 0
\end{array}\right] V^{*} \\
& =U\left[\begin{array}{cc}
\left(W_{1} A_{1} W_{1}\right)^{-1} & \left(A_{1} W_{1}\right)^{-2}\left(A_{12}+W_{1}^{-1} W_{12} A_{2}\right) \\
0 & 0
\end{array}\right] V^{*} .
\end{aligned}
$$


Now, we shall prove that the matrix $X$ satisfies the system (12). In fact, setting $S_{A W}:=A_{1} W_{12}+A_{12} W_{2}$ and $G:=A_{12}+W_{1}^{-1} W_{12} A_{2}$ we get

$$
\begin{aligned}
& X W X=U\left[\begin{array}{cc}
\left(A_{1} W_{1}\right)^{-1} & \left(W_{1} A_{1} W_{1}\right)^{-1} W_{12}+\left(A_{1} W_{1}\right)^{-2} G W_{2} \\
0 & 0
\end{array}\right]\left[\begin{array}{cc}
\left(W_{1} A_{1} W_{1}\right)^{-1} & \left(A_{1} W_{1}\right)^{-2} G \\
0 & 0
\end{array}\right] V^{*} \\
& =U\left[\begin{array}{cc}
\left(A_{1} W_{1}\right)^{-2} W_{1}^{-1} & \left(A_{1} W_{1}\right)^{-3} G \\
0 & 0
\end{array}\right] V^{*},
\end{aligned}
$$

and so,

$$
\begin{aligned}
& A \star X^{\star 2}=A W X W X=U\left[\begin{array}{cc}
A_{1} W_{1} & S_{A W} \\
0 & A_{2} W_{2}
\end{array}\right]\left[\begin{array}{cc}
\left(A_{1} W_{1}\right)^{-2} W_{1}^{-1} & \left(A_{1} W_{1}\right)^{-3} G \\
0 & 0
\end{array}\right] V^{*} \\
& =U\left[\begin{array}{cc}
\left(A_{1} W_{1}\right)^{-1} W_{1}^{-1} & \left(A_{1} W_{1}\right)^{-2} G \\
0 & 0
\end{array}\right] V^{*}=U\left[\begin{array}{cc}
\left(W_{1} A_{1} W_{1}\right)^{-1} & \left(A_{1} W_{1}\right)^{-2} G \\
0 & 0
\end{array}\right] V^{*}=X .
\end{aligned}
$$

Also, we have

$$
\begin{aligned}
A \star X & =A W X=U\left[\begin{array}{cc}
A_{1} W_{1} & S_{A W} \\
0 & A_{2} W_{2}
\end{array}\right]\left[\begin{array}{cc}
\left(W_{1} A_{1} W_{1}\right)^{-1} & \left(A_{1} W_{1}\right)^{-2} G \\
0 & 0
\end{array}\right] V^{*} \\
& =U\left[\begin{array}{cc}
W_{1}^{-1} & \left(A_{1} W_{1}\right)^{-1} G \\
0 & 0
\end{array}\right] V^{*}
\end{aligned}
$$

and

$$
\begin{aligned}
A^{\oplus, W} \star A & =A^{\oplus, W} W A=U\left[\begin{array}{cc}
\left(W_{1} A_{1} W_{1}\right)^{-1} & 0 \\
0 & 0
\end{array}\right]\left[\begin{array}{cc}
W_{1} A_{1} & W_{1} G \\
0 & W_{2} A_{2}
\end{array}\right] V^{*} \\
& =U\left[\begin{array}{cc}
W_{1}^{-1} & \left(A_{1} W_{1}\right)^{-1} G \\
0 & 0
\end{array}\right] V^{*} .
\end{aligned}
$$

From (13) and (14) we arrive at $A \star X=A^{\oplus, W} \star A$. Finally, the uniqueness given by Theorem 4 ensures that $A^{\oplus, W}=X$.

A direct consequence of the proof of Theorem 5 is given in the following corollary where the weighted weak group inverse is represented in terms of the weighted core EP inverse.

Corollary 2 If $A \in \mathbb{C}^{m \times n}$ then $A^{\circledast, W}=\left(A^{\oplus, W}\right)^{\star 2} \star A$.

Definition 3 Let $A \in \mathbb{C}^{m \times n}$ and $k=\max \{\operatorname{Ind}(A W)$, $\operatorname{Ind}(W A)\}$. The unique matrix $X \in \mathbb{C}^{m \times n}$ that satisfies the system (12) is called the $W$-weighted WG inverse of $A$.

As we have demonstrated, the matrix in Definition 3 is $X=A^{\circledR, W}$.

Remark 2 When $m=n$ and $W=I_{n}$, we recover the WG inverse using the representation given in (5).

Remark 3 When $k=1$, from the representations given in Theorem 3 and Theorem 5 , it is easy to verify that the weighted Drazin (group) inverse and the $W$-weighted WG inverse are coincide.

This new inverse is different from the known ones as the following example shows. Thus, the $W$-weighted WG inverses provide a new class of generalized inverses for rectangular matrices. 
Example 1 For the matrices

$$
A=\left[\begin{array}{lll}
1 & 0 & 0 \\
0 & 0 & 1
\end{array}\right] \quad \text { and } \quad W=\left[\begin{array}{ll}
1 & 1 \\
1 & 0 \\
0 & 0
\end{array}\right],
$$

we have $k=\max \{1,2\}=2$. The weighted Drazin inverse, the weighted core EP inverse, and the $W$-weighted WG inverse are

$$
A^{d, W}=\left[\begin{array}{lll}
1 & 0 & 1 \\
0 & 0 & 0
\end{array}\right], \quad A^{\oplus, W}=\left[\begin{array}{ccc}
\frac{1}{2} & \frac{1}{2} & 0 \\
0 & 0 & 0
\end{array}\right], \quad \text { and } \quad A^{\circledR, W}=\left[\begin{array}{ccc}
1 & 0 & \frac{1}{2} \\
0 & 0 & 0
\end{array}\right],
$$

the Moore-Penrose inverse, the weighted DMP inverse and the weighted CMP inverse are

$$
A^{\dagger}=\left[\begin{array}{ll}
1 & 0 \\
0 & 0 \\
0 & 1
\end{array}\right], \quad A^{d, \dagger, W}=\left[\begin{array}{ll}
1 & 1 \\
1 & 1 \\
0 & 0
\end{array}\right], \quad \text { and } \quad A^{c, \dagger, W}=\left[\begin{array}{ll}
1 & 1 \\
0 & 0 \\
0 & 0
\end{array}\right] .
$$

Clearly, these kind of inverses could have change to be the same only for $m=n$, that is, if $m \neq n$ the $A^{d, W}, A^{\oplus, W}$, and $A^{\circledR, W}$ never coincide with $A^{\dagger}, A^{d, \dagger, W}$, and $A^{c, \dagger, W}$.

\section{Representations and characterizations of the $W$-weighted WG inverse}

This section is devoted to go further into the analysis of $W$-weighted WG inverses.

It is well known that for a square matrix $A$, in general, $\left(A^{\dagger}\right)^{2} \neq\left(A^{2}\right)^{\dagger}$ (that is, $A$ is not bi-dagger). However, for the weighted core EP inverse the property is true.

Lemma 1 Let $A \in \mathbb{C}^{m \times n}$. Then $\left(A^{\oplus, W}\right)^{\star 2}=\left(A^{\star 2}\right)^{\oplus, W}$.

Proof Using the weighted core EP decomposition (7) of the pair $\{A, W\}$, in the proof of Theorem 5 we have shown the equality

$$
\left(A^{\oplus, W}\right)^{\star 2}=U\left[\begin{array}{cr}
\left(W_{1} A_{1} W_{1}\right)^{-1}\left(W_{1} A_{1}\right)^{-1} & 0 \\
0 & 0
\end{array}\right] V^{*} .
$$

On the other hand,

$$
A^{\star 2}=A \star A=A W A=U\left[\begin{array}{cc}
A_{1} W_{1} A_{1} & A_{1} W_{1} A_{12}+\left(A_{1} W_{12}+A_{12} W_{2}\right) A_{2} \\
0 & A_{2} W_{2} A_{2}
\end{array}\right] V^{*} .
$$

That is, what we have really obtained in (16) is a weighted core EP decomposition as in (7) of the pair $\left\{A^{\star 2}, W\right\}$. Then, it is not hard to see that the weighted core EP inverse of $A^{\star 2}$ is obtained via the expression (8) and it coincides with (15), that is, $\left(A^{\oplus, W}\right)^{\star 2}=\left(A^{\star 2}\right)^{\oplus, W}$.

Remark 4 Rewriting Lemma 1 as $(A W A)^{\oplus, W}=A^{\oplus, W} W A^{\oplus, W}$, the bi-weigthed core EP inverse property can be interpreted as a reverse order law of a triple.

Based on Corollary 2 and Lemma 1 we have the following result. 
Theorem 6 Let $A \in \mathbb{C}^{m \times n}$. Then $A^{\circledR, W}=\left(A^{\oplus, W}\right)^{\star 2} \star A=\left(A^{\star 2}\right)^{\oplus, W} \star A$.

In the following theorem we establish another way to compute the $W$-weighted WG inverse by using the weighted group inverse. It can be really seen as a representation of the $W$-weighted WG inverse in terms of the weighted core EP inverse and the weighted group inverse.

Theorem 7 Let $A \in \mathbb{C}^{m \times n}$. Then

$$
A^{\circledR, W}=\left(A \star A^{\oplus, W} \star A\right)^{\#, W} .
$$

Proof From the weighted core EP decomposition of the pair $\{A, W\}$ given in (7) and from the expression for the weighted core EP inverse given in (8) we have

$$
\begin{aligned}
B & :=A \star A^{\oplus, W} \star A=A W A^{\oplus, W} W A \\
& =U\left[\begin{array}{cc}
A_{1} W_{1} & A_{1} W_{12}+A_{12} W_{2} \\
0 & A_{2} W_{2}
\end{array}\right]\left[\begin{array}{cc}
\left(W_{1} A_{1} W_{1}\right)^{-1} & 0 \\
0 & 0
\end{array}\right]\left[\begin{array}{cc}
W_{1} A_{1} & W_{1} A_{12}+W_{12} A_{2} \\
0 & W_{2} A_{2}
\end{array}\right] V^{*} \\
& =U\left[\begin{array}{cc}
A_{1} & A_{12}+W_{1}^{-1} W_{12} A_{2} \\
0 & 0
\end{array}\right] V^{*} .
\end{aligned}
$$

We observe that

$$
W B=V\left[\begin{array}{cc}
W_{1} A_{1} & W_{1} A_{12}+W_{12} A_{2} \\
0 & 0
\end{array}\right] V^{*}
$$

whence we arrive at the conclusion that $\operatorname{Ind}(W B)=1$. Similarly, since

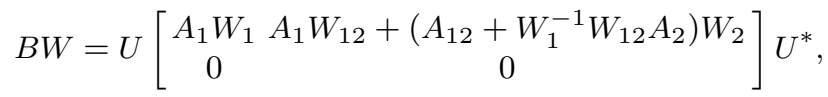

we get $\operatorname{Ind}(B W)=1$. Therefore, $\max \{\operatorname{Ind}(B W), \operatorname{Ind}(W B)\}=1$. Now, applying Corollary 1 to the matrix $B$ we obtain

$$
\left(A \star A^{\oplus, W} \star A\right)^{\#, W}=U\left[\begin{array}{cc}
\left(W_{1} A_{1} W_{1}\right)^{-1} & \left(A_{1} W_{1}\right)^{-2}\left(A_{12}+W_{1}^{-1} W_{12} A_{2}\right) \\
0 & 0
\end{array}\right] V^{*} .
$$

Finally, Theorem 5 completes the proof.

By using the unitary matrix $U$ found in (7) corresponding to the pair $\{A, W\}$ and [10, Lemma 2.5], we consider the following orthogonal projector

$$
P_{A^{\star k} \star I_{m}}=P_{(A W)^{k}}=(A W)^{k}\left[(A W)^{k}\right]^{\dagger}=U\left[\begin{array}{cc}
I_{\operatorname{rk}\left((A W)^{k}\right)} & 0 \\
0 & 0
\end{array}\right] U^{*} .
$$

Next, the $W$-weighted WG inverse can be expressed in terms of the Moore-Penrose inverse.

Theorem 8 Let $A \in \mathbb{C}^{m \times n}$ and $k=\max \{\operatorname{Ind}(A W), \operatorname{Ind}(W A)\}$. Then

$$
A^{®, W}=\left(I_{n} \star A^{\star 2} \star P_{\left(A^{\star k} \star I_{m}\right)}\right)^{\dagger} \star A .
$$


Proof From the weighted core EP decomposition (7) of the pair $\{A, W\}$ we have

$$
\begin{aligned}
& \left(I_{n} \star A^{\star 2} \star P_{\left(A^{\star k} \star I_{m}\right)}\right)^{\dagger} \star A=\left(W(A W)^{2} P_{(A W)^{k}}\right)^{\dagger} W A \\
& =\left(V\left[\begin{array}{cc}
W_{1}\left(A_{1} W_{1}\right)^{2} & G \\
0 & \left(W_{2} A_{2}\right)^{2} W_{2}
\end{array}\right]\left[\begin{array}{cc}
I_{\mathrm{rk}\left((A W)^{k}\right)} & 0 \\
0 & 0
\end{array}\right] U^{*}\right)^{\dagger} V\left[\begin{array}{c}
W_{1} A_{1} \\
W_{1} A_{12}+W_{12} A_{2} \\
0
\end{array}\right] W_{2} A_{2} \\
& =\left(V\left[\begin{array}{cc}
W_{1}\left(A_{1} W_{1}\right)^{2} & 0 \\
0 & 0
\end{array}\right] U^{*}\right)^{\dagger} V\left[\begin{array}{cc}
W_{1} A_{1} & W_{1} A_{12}+W_{12} A_{2} \\
0 & W_{2} A_{2}
\end{array}\right] V^{*} \\
& =U\left[\begin{array}{cc}
\left(A_{1} W_{1}\right)^{-2} W_{1}^{-1} & 0 \\
0 & 0
\end{array}\right]\left[\begin{array}{cc}
W_{1} A_{1} & W_{1} A_{12}+W_{12} A_{2} \\
0 & W_{2} A_{2}
\end{array}\right] V^{*} \\
& =U\left[\begin{array}{cc}
\left(W_{1} A_{1} W_{1}\right)^{-1} & \left(A_{1} W_{1}\right)^{-2}\left(A_{12}+W_{1}^{-1} W_{12} A_{2}\right) \\
0 & 0
\end{array}\right] V^{*},
\end{aligned}
$$

for some matrix $G$. Finally, Theorem 5 completes the proof.

Since the weighted Drazin inverse and the $W$-weighted WG inverse are two generalizations of the weighted group inverse, next we will see the similarities and differences between them.

Next result gives a property of the $W$-weighted weak group inverse similar to that satisfied by the weighted Drazin inverse (see Remark 5 below).

Theorem 9 Let $A \in \mathbb{C}^{m \times n}$ and $k=\max \{\operatorname{Ind}(A W), \operatorname{Ind}(W A)\} \geq 1$. Then

$$
A^{\circledR, W} \star A^{\star(k+1)} \star I_{m}=A^{\star k} \star I_{m} .
$$

Proof We observe that $A^{\circledR, W} \star A^{\star(k+1)} \star I_{m}=A^{\star k} \star I_{m}$ is equivalent to the equality $A^{\circledR, W} W(A W)^{k+1}=(A W)^{k}$. Setting $S_{A W}:=A_{1} W_{12}+A_{12} W_{2}$ and recalling the expression for $\widetilde{T}_{A W}$ in the proof of Theorem 3 we get

$$
\widetilde{T}_{A W}=\sum_{j=0}^{k-1}\left(A_{1} W_{1}\right)^{j-k-1} S_{A W}\left(A_{2} W_{2}\right)^{k-1-j} .
$$

Thus,

$$
(A W)^{k}=U\left[\begin{array}{cc}
\left(A_{1} W_{1}\right)^{k} & \widetilde{T}_{A W} \\
0 & 0
\end{array}\right] U^{*}
$$

and

$$
(A W)^{k+1}=U\left[\begin{array}{cc}
\left(A_{1} W_{1}\right)^{k+1} & \left(A_{1} W_{1}\right)^{k} S_{A W}+\widetilde{T}_{A W} A_{2} W_{2} \\
0 & 0
\end{array}\right] U^{*} .
$$

Also, by Theorem 5 we have

$$
A^{\circledR, W} W=U\left[\begin{array}{cc}
\left(A_{1} W_{1}\right)^{-1} & G \\
0 & 0
\end{array}\right] U^{*},
$$

for some matrix $G$. Now, from (20) and (21) we obtain

$$
A^{\circledR, W} W(A W)^{k+1}=U\left[\begin{array}{cc}
\left(A_{1} W_{1}\right)^{k} & \left(A_{1} W_{1}\right)^{k-1} S_{A W}+\left(A_{1} W_{1}\right)^{-1} \widetilde{T}_{A W} A_{2} W_{2} \\
0 & 0
\end{array}\right] U^{*} .
$$

After a little algebra, it is not hard to check that

$$
\widetilde{T}_{A W}=\left(A_{1} W_{1}\right)^{k-1} S_{A W}+\left(A_{1} W_{1}\right)^{-1} \widetilde{T}_{A W} A_{2} W_{2} .
$$

Consequently, the equality in (18) follows from (19) and (22). 
Remark 5 The condition $X W(A W)^{k+1}=(A W)^{k}$ in the Definition 1 says that $X W$ is a weak Drazin inverse of $A W$ (see [5, Definition 9.7.1, p. 203]). In a similar way, the expression (18) in Theorem 9 can be phrased saying that the $W$-weighted WG inverse $A^{\circledR, W}$ of $A$ is a weak Drazin inverse of $A$ with respect to the $W$-product of the Banach algebra $\left(\mathbb{C}^{m \times n}, \star,\|\cdot\|_{W}\right)$.

For a square matrix $A$ of index $k$, a useful representation of $A^{d}$ by means of the group inverse is given by $A^{d}=A^{k}\left(A^{k+1}\right)^{\#}[1$, Lemma 5, p. 154]. For weak group inverses (of square matrices), a similar result involving the core inverse was stated. More precisely, in [25, Theorem 3.4] the equality $A^{\circledR}=A^{k}\left(A^{k+2}\right)^{\oplus} A$ was derived.

The following theorem is an natural extension of this last identity.

Theorem 10 Let $A \in \mathbb{C}^{m \times n}$ and $k=\max \{\operatorname{Ind}(A W), \operatorname{Ind}(W A)\}$. Then

$$
A^{\circledast, W}=A^{\star k} \star\left(A^{\star(k+2)}\right)^{\oplus, W} \star A .
$$

Proof We consider the weighted core EP decomposition of the pair $\{A, W\}$ given in (7). From (19), (20), and the inverse computed in (8) (observe that $(A W)^{k+1} A$ has index at most 1$)$, we get

$$
\begin{aligned}
& A^{\star k} \star\left[\left(A^{\star(k+2)}\right]^{\boxplus, W} \star A=(A W)^{k}\left[(A W)^{k+1} A\right]^{\oplus, W} W A\right. \\
& =U\left[\begin{array}{cc}
\left(A_{1} W_{1}\right)^{k} & \widetilde{T}_{A W} \\
0 & 0
\end{array}\right]\left[\begin{array}{cc}
{\left[W_{1}\left(A_{1} W_{1}\right)^{k+2}\right]^{-1}} & 0 \\
0 & 0
\end{array}\right]\left[\begin{array}{cc}
W_{1} A_{1} & W_{1} A_{12}+W_{12} A_{2} \\
0 & W_{2} A_{2}
\end{array}\right] V^{*} \\
& =U\left[\begin{array}{cc}
\left(A_{1} W_{1}\right)^{-2} W_{1}^{-1} & 0 \\
0 & 0
\end{array}\right]\left[\begin{array}{cc}
W_{1} A_{1} & W_{1} A_{12}+W_{12} A_{2} \\
0 & W_{2} A_{2}
\end{array}\right] V^{*} \\
& =U\left[\begin{array}{cc}
\left(W_{1} A_{1} W_{1}\right)^{-1} & \left(A_{1} W_{1}\right)^{-2}\left(A_{12}+W_{1}^{-1} W_{12} A_{2}\right) \\
0 & 0
\end{array}\right] V^{*} .
\end{aligned}
$$

Now, Theorem 5 completes the proof.

It is well known that $\mathcal{R}\left(A^{d, W}\right)=\mathcal{R}\left((A W)^{k}\right)$ holds (see, for example, [26, Lemma 2]). It turns out that this equality remains valid also when the weighted Drazin inverse is replaced with the $W$-weighted WG inverse.

Theorem 11 Let $A \in \mathbb{C}^{m \times n}$ and $k=\max \{\operatorname{Ind}(A W), \operatorname{Ind}(W A)\}$. Then

$$
\mathcal{R}\left(A^{\circledR, W}\right)=\mathcal{R}\left(A^{d, W}\right)=\mathcal{R}\left(A^{\star k} \star I_{m}\right)=\mathcal{R}\left(A \star A^{\circledR, W}\right) .
$$

Proof By Theorem $5, A^{\circledR, W}=A^{\oplus, W} \star A^{\oplus, W} \star A$ holds and then $\mathcal{R}\left(A^{\circledR, W}\right) \subseteq$ $\mathcal{R}\left(A^{\oplus, W}\right) \subseteq \mathcal{R}\left((A W)^{k}\right)$, where the last inclusion is due to Definition 2 . By Theorem 9 , the converse inclusion $\mathcal{R}\left((A W)^{k}\right) \subseteq \mathcal{R}\left(A^{\circledR, W}\right)$ holds. Therefore, $\mathcal{R}\left(A^{\circledR, W}\right)=$ $\mathcal{R}\left(A^{d, W}\right)=\mathcal{R}\left((A W)^{k}\right)=\mathcal{R}\left(A^{\star k} \star I_{m}\right)$.

It remains to show the equality $\mathcal{R}\left(A \star A^{\circledR, W}\right)=\mathcal{R}\left((A W)^{k}\right)$. Since by definition $A \star A^{\circledR, W}=A^{\oplus, W} \star A$ holds, we obtain the inclusions $\mathcal{R}\left(A \star A^{\circledR, W}\right) \subseteq \mathcal{R}\left(A^{\oplus, W}\right) \subseteq$ $\mathcal{R}\left((A W)^{k}\right)$. According to [10, Theorem 5.7], we have $A^{\oplus, W} \star A^{\star(k+2)}=A^{\star(k+1)}$, which implies

$\mathcal{R}\left((A W)^{k}\right)=\mathcal{R}\left((A W)^{k+1}\right) \subseteq \mathcal{R}\left((A W)^{k} A\right)=\mathcal{R}\left(A^{\star(k+1)}\right) \subseteq \mathcal{R}\left(A^{\oplus, W} \star A\right)=\mathcal{R}\left(A \star A^{\bowtie, W}\right)$.

Consequently, $\mathcal{R}\left(A \star A^{\circledast, W}\right)=\mathcal{R}\left((A W)^{k}\right)=\mathcal{R}\left(A^{\star k} \star I_{m}\right)$. This complete the proof. 
On the other hand, it is known that $\left(A^{d, W}\right)^{\star 2}=\left(A^{\star 2}\right)^{d, W}[7$, Proposition 4.1] but this property is not always true for the $W$-weighted WG inverses.

Next result characterizes when the aforementioned property holds.

Corollary 3 Let $A \in \mathbb{C}^{m \times n}$ and consider the weighted core EP decomposition of the pair $\{A, W\}$ as in (7). Then $\left(A^{\star 2}\right)^{\circledR, W}=\left(A^{\circledR, W}\right)^{\star 2}$ if and only if $\left(W_{1} A_{12}+\right.$ $\left.W_{12} A_{2}\right) W_{2} A_{2}=0$.

Proof From (16) we get a weighted core EP decomposition of the pair $\left\{A^{\star 2}, W\right\}$. Now, by using Theorem 5 we obtain

$$
\left(A^{\star 2}\right)^{\circledR, W}==U\left[\begin{array}{cc}
W_{1}^{-1}\left(W_{1} A_{1}\right)^{-2} & \left(A_{1} W_{1}\right)^{-4} G \\
0 & 0
\end{array}\right] V^{*},
$$

where $G=A_{1} W_{1} A_{12}+\left(A_{1} W_{12}+A_{12} W_{2}\right) A_{2}+W_{1}^{-1} W_{12} A_{2} W_{2} A_{2}$, and

$$
\left(A^{\circledast, W}\right)^{\star 2}=A^{\circledast, W} \star A^{\circledast, W}=U\left[\begin{array}{c}
W_{1}^{-1}\left(W_{1} A_{1}\right)^{-2}\left(A_{1} W_{1}\right)^{-3}\left(A_{12}+W_{1}^{-1} W_{12} A_{2}\right) \\
0
\end{array}\right] V^{*} .
$$

Consequently, $\left(A^{\star 2}\right)^{\circledR, W}=\left(A^{\circledR, W}\right)^{\star 2}$ if and only if

$$
\left(A_{1} W_{1}\right)^{-4} G=\left(A_{1} W_{1}\right)^{-3}\left(A_{12}+W_{1}^{-1} W_{12} A_{2}\right) .
$$

After a little algebra, we get that the above equation is equivalent to

$$
\left(A_{12}+W_{1}^{-1} W_{12} A_{2}\right) W_{2} A_{2}=0 .
$$

From Definition 1 it is known that $A \star A^{d, W}=A^{d, W} \star A$ must be always fulfilled. It is of interest to inquire whether the same property is true or not for the $W$-weighted WG inverse.

Corollary 4 Let $A \in \mathbb{C}^{m \times n}$ and consider the weighted core EP decomposition of the pair $\{A, W\}$ as in (7). Then $A \star A^{\circledR, W}=A^{\circledR, W} \star A$ if and only if $\left(W_{1} A_{12}+\right.$ $\left.W_{12} A_{2}\right) W_{2} A_{2}=0$.

Proof From the weighted core EP decomposition of the pair $\{A, W\}$ given in (7) and Theorem 5 we have

$$
A \star A^{\circledR, W}=U\left[\begin{array}{cc}
W_{1}^{-1} & \left(A_{1} W_{1}\right)^{-1}\left(A_{12}+W_{1}^{-1} W_{12} A_{2}\right) \\
0 & 0
\end{array}\right] V^{*}
$$

and

$$
A^{\circledR, W} \star A=U\left[\begin{array}{cc}
W_{1}^{-1} & \left(W_{1} A_{1} W_{1}\right)^{-1}\left(W_{1} A_{12}+W_{12} A_{2}\right)+F \\
0 & 0
\end{array}\right] V^{*},
$$

where $F=\left(A_{1} W_{1}\right)^{-2}\left(A_{12}+W_{1}^{-1} W_{12} A_{2}\right) W_{2} A_{2}$.

Therefore, $A \star A^{\circledR, W}=A^{\circledR, W} \star A$ if and only if

$$
\left(A_{1} W_{1}\right)^{-1}\left(A_{12}+W_{1}^{-1} W_{12} A_{2}\right)=\left(W_{1} A_{1} W_{1}\right)^{-1}\left(W_{1} A_{12}+W_{12} A_{2}\right)+F,
$$

which is equivalent to $\left(W_{1} A_{12}+W_{12} A_{2}\right) W_{2} A_{2}=0$.

The following result can be easily derived from Corollary 3 and Corollary 4 . 
Corollary 5 Let $A \in \mathbb{C}^{m \times n}$. Then

$$
\left(A^{\star 2}\right)^{\circledR, W}=\left(A^{\circledR, W}\right)^{\star 2} \text { if and only if } A \star A^{\circledR, W}=A^{\circledR, W} \star A .
$$

Sufficient conditions for $A^{\circledR, W}=A^{d, W}$ to be true are established in the two subsequent corollaries.

Corollary 6 Let $A \in \mathbb{C}^{m \times n}$ such that $A \star A^{\circledR, W}=A^{\circledR, W} \star A$. Then $A^{\circledR, W}=A^{d, W}$.

Proof From the weighted core EP decomposition of the pair $\{A, W\}$ given in $(7)$ and Corollary 4 we have that $\left(W_{1} A_{12}+W_{12} A_{2}\right) W_{2} A_{2}=0$. Therefore, from Theorem 3 we have

$$
\begin{aligned}
A^{d, W} & =U\left[\begin{array}{cc}
\left(W_{1} A_{1} W_{1}\right)^{-1} & A_{1}\left(W_{1} A_{1}\right)^{-3}\left(W_{1} A_{12}+W_{12} A_{2}\right) \\
0 & 0
\end{array}\right] V^{*} \\
& =U\left[\begin{array}{cc}
\left(W_{1} A_{1} W_{1}\right)^{-1} & \left(A_{1} W_{1}\right)^{-2}\left(A_{12}+W_{1}^{-1} W_{12} A_{2}\right) \\
0 & 0
\end{array}\right] V^{*} .
\end{aligned}
$$

Finally, from (23) and Theorem 5 it follows that $A^{\circledR, W}=A^{d, W}$.

Corollary 7 Let $W \in \mathbb{C}^{n \times m}$ be a nonzero matrix and $A \in \mathbb{C}^{m \times n}$ satisfying $\left(A^{\star 2}\right)^{\circledR, W}$ $\left(A^{\circledR, W}\right)^{\star 2}$. Then $A^{\circledR, W}=A^{d, W}$.

Proof It is a direct application of Corollary 5 and Corollary 6.

From (8) and Theorem 5 we can establish the following result.

Corollary 8 Let $A \in \mathbb{C}^{m \times n}$ and consider the weighted core EP decomposition of the pair $\{A, W\}$ as in (7). Then $A^{\circledR, W}=A^{\oplus, W}$ if and only if $W_{1} A_{12}+W_{12} A_{2}=0$.

Finally, the validity of the condition $W_{1} A_{12}+W_{12} A_{2}=0$ implies the equality of the three classes of inverses, that is

$$
A^{®, W}=A^{\oplus, W}=A^{d, W} .
$$

\section{Acknowledgements}

We woud like to thank the Referees for carefully reading our manuscript and for giving constructive comments which susbtancially helped improving the quality of the paper.

\section{References}

1. Ben-Israel, A., Greville, T.N.E.: Generalized Inverses: Theory and Applications. Second Ed., Springer-Verlag, New York (2003)

2. Baksalary, O.M., Trenkler, G.: Core inverse of matrices. Linear Multilinear Algebra, 58, 681-697 (2010)

3. Baksalary, O.M., Trenkler, G.: On a generalized core inverse. Appl. Math. Comput., 236, 450-457 (2014)

4. Bajodah, A.H.: Servo-constraint generalized inverse dynamics for robot manipulator control design. International Journal on Robotics and Automation, 25 (2010). http://doi.org/10.2316/Journal.206.2016.1.206-3291 
5. Campbell, S.L., Meyer Jr., C.D.: Generalized Inverses of Linear transformations. SIAM, Philadelphia (2009)

6. Cline, R.E., Greville, T.N.E.: A Drazin inverse for rectangular matrices. Linear Algebra Appl., 29, 53-62 (1980)

7. Dajić, A., Koliha, J.J.: The weighted g-Drazin inverse for operators. J. Aust. Math. Soc., 2, 163-181 (2007)

8. Doty, K.L., Melchiorri, C., Bonivento, C.: A Theory of Generalized Inverses Applied to Robotics. Int. J. Rob. Res., 12, 1-19 (1993)

9. Drazin, M.P.: Pseudo-inverses in associate rings and semirings. Amer. Math. Monthly, 65, 506-514 (1958)

10. Ferreyra, D.E., Levis, F.E., Thome, N.: Revisiting of the core EP inverse and its extension to rectangular matrices. Quaest. Math., 41, 265-281 (2018)

11. Ferreyra, D.E., Levis, F.E., Thome, N.: Maximal classes of matrices determining generalized inverses. Appl. Math. Comput., 333, 42-52 (2018)

12. Gigola, S., Lebtahi, L., Thome, N.: The inverse eigenvalue problem for a Hermitian reflexive matrix and the optimization problem. J. Comput. Appl. Math., 291, 449-457 (2016)

13. Hartwig, R.E.: The weighted *-core-nilpotent decomposition. Linear Algebra Appl., 211, 101-111 (1994)

14. Kirkland, S.J., Neumann, M.: Group Inverses of M-Matrices and Their Applications. Chapman and Hall/CRC, London (2013)

15. Malik, S., Thome, N.: On a new generalized inverse for matrices of an arbitrary index. Appl. Math. Comput., 226, 575-580 (2014)

16. Malešević, B., Obradović, R., Banjac, B., Jovović, I., Makragić, M.: Application of polynomial texture mapping in process of digitalization of cultural heritage. https://arxiv.org/pdf/1312.6935 (2013). Accessed 14 June 2018

17. Manjunatha Prasad, K., Mohana, K.S.: Core EP inverse. Linear Multilinear Algebra, 62, 792-802 (2014)

18. Mehdipour, M. Salemi, A.: On a new generalized inverse of matrices. Linear Multilinear Algebra, 66, 1046-1053 (2018)

19. Meng, L.S.: The DMP inverse for rectangular matrices. Filomat, 31, 6015-6019 (2017)

20. Mosić, D.: The CMP inverse for rectangular matrices. Aequaetiones Math., 92, 649-659 (2018)

21. Penrose, R.: A generalized inverse for matrices. Proceedings of the Cambridge Philosophical Society, 51, 406-413 (1955)

22. Soleimani, F., Stanimirović, P.S., Soleymani, F.: Some Matrix Iterations for Computing Generalized Inverses and Balancing Chemical Equations. Algorithms, 8, 982-998 (2015)

23. Xiao, G.Z., Shen, B.Z., Wu, C.K., Wong, C.S.: Some spectral techniques in coding theory. Discrete Math., 87, 181-186 (1991)

24. Wang, H.: Core-EP decomposition and its applications. Linear Algebra Appl., 508, 289-300 (2016)

25. Wang, H., Chen, J.: Weak group inverse. Open Math., 16, 1218-1232, (2018)

26. Wei, Y.: A characterization for the $W$-weighted Drazin inverse and a Crammer rule for the $W$-weighted Drazin inverse solution. Appl. Math. Comput., 125, 303-310, (2002) 